\title{
La gestión del infoconocimiento en instituciones de educación superior ${ }^{1}$
}

\author{
Marisleidy Alba Cabañas ${ }^{2}$ \\ Liliana Gutiérrez de Piñeres Botero ${ }^{3}$
}

\begin{abstract}
Alba, Cabañas M., y Gutiérrez de Piñeres Botero, L. (2020). La gestión del infoconocimiento en instituciones de Educación Superior. Revista Activos, 18(1), 95-122. DoI: https://doi.org/10.15332/25005278/6160
\end{abstract}

Recibido: 12 de octubre de 2019

Aprobado: 06 de noviembre de 2019

\section{Clasificación JEL: M1, M12, M15, M54}

1 Resultado de consultoría asociada al proyecto de investigación sobre flujos de información y conocimiento del grupo en Sistemas de Información y Control Organizacional de la Facultad de Contaduría Pública de la Universidad Externado de Colombia.

2 Ph. D. en Ciencias Económicas, coordinadora de investigación de la Facultad de Contaduría Pública de la Universidad Externado de Colombia. e-mail: marisleidy. alba@uexternado.edu.co ORCID: https://orcid.org/0000-0001-6935-6097

3 MBA, Directora Académica y de Investigación de la Facultad de Contaduría Pública de la Universidad Externado de Colombia. liliana.gutierrezdepineres@ uexternado.edu.co

ORCID: https://orcid.org/0000-0001-6778-707X 


\section{Resumen}

Las instituciones de educación superior muestran algunas debilidades y problemáticas en los procedimientos, la protección y evaluación de la información, en las formas para evitar la pérdida, difusión de información sensible, y en la conservación del conocimiento. A esto se suma la obligación de responder a los planes de mejoramiento, a los nuevos sistemas de información, a eliminar la burocracia, las duplicidades y a eliminar errores y pérdidas de información. Estas son las dificultades que han llevado a intervenir la gestión de procesos de estas instituciones.

Siguiendo las tipologías de consultoría gerencial, a través de una metodología de diagnóstico se estudiaron los procesos, los puntos críticos, la gestión de la información y el estado del proceso de gestión del conocimiento. El resultado de esta mejora permite construir un modelo de gestión del infoconocimiento, así como un procedimiento en el que se identifican los pasos para desarrollar esta propuesta.

Palabras clave: conservación del conocimiento, consultoría gerencial, gestión de la información, protección de la información. 


\title{
Info-knowledge Management in Higher Education Institutions
}

\begin{abstract}
Higher education institutions show some weaknesses and problems in the processing, protection, and evaluation of information, as well as in the procedures to avoid loss or dissemination of sensitive information and to preserve knowledge. In addition, there is the obligation to respond to improvement plans, build new information systems, eliminate bureaucracy and duplication, and to prevent errors and information loss. These are the difficulties that have led to the intervention of the process management of these institutions.

The processes and critical points of information management and the status of knowledge management procedures were analyzed following the typology of management consulting and using a diagnostic methodology. The result of this improvement makes it possible to build an info-knowledge management model, as well as a procedure in which the steps to develop this proposal are identified.
\end{abstract}

Keywords: knowledge preservation, management consulting, information management, information protection. 


\section{Introducción}

La información, el conocimiento y su gestión se ha convertido en una opción estratégica de la organización para alcanzar los objetivos y metas. Por ello, en el contexto de la globalización y las transformaciones, la gestión del potencial del conocimiento es una herramienta eficaz para aumentar la eficacia de las organizaciones. En este sentido, distinguir los principales procesos de gestión del conocimiento, los recursos de la organización y las oportunidades de mercado se ha convertido en un objetivo, sobre todo si se quiere satisfacer a los usuarios.

El entorno actual se caracteriza por la necesidad de introducir el conocimiento en las actividades que se desarrollan. Las organizaciones reconocen que los factores de conocimiento tienen un impacto significativo o valor agregado en el resultado de las empresas. La existencia, la supervivencia a largo plazo y la rentabilidad depende de la competitividad de los productos y servicios. La transformación de "materias primas" en productos competitivos solo es posible con el conocimiento de los involucrados. Por lo tanto, es necesario identificar los factores de conocimiento que pueden influir en un resultado positivo de las organizaciones.

Las Instituciones de Educación Superior (IES) no están ajenas a esta necesidad, pues, como entes creadores de conocimiento, necesitan implementar acciones que garanticen los procesos de identificación, socialización, almacenamiento, transferencia y creación de conocimiento. El conocimiento entonces, dentro de las IES, se convierte en el elemento primordial para su actuar, por lo que su producción y manejo garantizaría adecuadamente el cumplimiento de su razón de ser (Caicedo, 2010).

Un estudio de las aportaciones de De Freitas y Oltra (2014), Alba y Herrera (2016), Topete et ál. (2012), Angulo y Negrón (2008), GarcíaPeñalvo (2016), y Martínez y Sánchez Muñoz (2018) demuestra que la dinámica de las IEs no dista de las problemáticas de cualquier tipo de organización empresarial, en donde se destacan debilidades en los procedimientos, en la protección y evaluación de la información, en las formas 
para evitar la pérdida, difusión de información sensible y la conservación del conocimiento. Allí se dejan a un lado los conocimientos tácitos existentes en el capital humano de las organizaciones y se descuida la información que no queda documentada a cual puede influir en el desarrollo de experiencia y en la identificación de necesidades organizacionales. Asimismo, se desconoce cómo las organizaciones deben determinar el conocimiento, bajo qué vías, métodos o infraestructura, es decir, si se debe realizar en informes o implementar herramientas tecnológicas (Alba, 2020).

La gestión del conocimiento sigue siendo imprecisa y deja a la espontaneidad un proceso que debería ser planificado, organizado y controlado, pues expresa que los conocimientos deberían ponerse a disposición en la medida en que sea necesario.

Atendiendo a los resultados encontrados anteriormente, es necesario realizar una revisión de los términos knowledge management y higher education. Para ello, desde la base de datos Scopus, se seleccionaron los años 2018, 2019 y 2020, y se recuperaron documentos con sus autores, resúmenes, volumen, número al que pertenecen y un total de 5583 palabras clave. De ellas, se seleccionan 300 que alcanzan el umbral. Para el análisis de la información se utilizó la herramienta VOSviewer a través de los métodos de estudio de la ocurrencia y la citación (figura 1).

Por medio de la ocurrencia y a través del esquema de clusterización se analizaron las 300 palabras clave. El resultado generó 9 agrupaciones temáticas, en las que se reflejó el grado de similitud de las palabras clave. Las agrupaciones temáticas resultantes se visualizaron a través de un mapa bibliométrico de red (figura 1), en el cual el tamaño de los clústeres estuvo determinado por diferentes factores, tales como el número de palabras clave dentro de los clústeres, frecuencia de ocurrencias de las palabras clave, y su peso o índice similitud. Cada grupo o clúster se distinguió por un color aleatorio. Los clústeres situados en el centro del mapa indicaron una alta interrelación de las palabras clave que los conformaron, mientras que los clústeres situados en los márgenes o bordes del mapa indicaron una menor. 
Así las cosas, como se determina en la figura 1, cuando se habla de knowledge management y higher education, se observa una fuerte relación con las siguientes líneas: compartir conocimiento, competencias, sistemas de aprendizaje en línea, desarrollo sostenible, entrenamiento personal, sistemas inteligentes, e instrumentos para la evaluación académica e innovación. Estas líneas están dirigidas a los procesos de aprendizaje y no muestran una vinculación de la gestión del conocimiento organizacional que debe estar presente en todas las IEs.

Figura 1. Mapa bibliométrico de ocurrencia de palabras clave. Knowledge management y higher education.

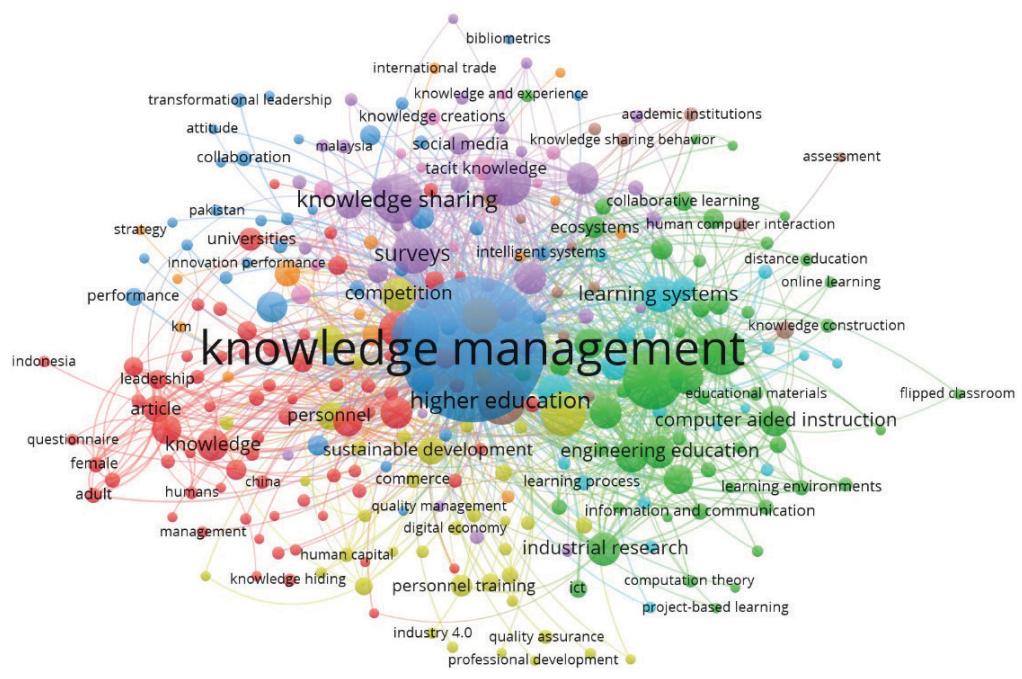

Fuente: elaboración propia a partir de Scopus, 2020 y VOSViewer versión 1.6.13.

Por otro lado, y a través de una revisión de autores con mayor citación en los temas de knowledge management y higher education entre los que se encuentran: Doulik et ál. (2017), Simonova y Poulova (2016), Veer-Ramjeawon y Rowley (2019), Alhabeeb y Rowley (2018), Pal Pandi et ál. (2018); Gafurov et ál. (2020); Safiullin y Akhmetshin (2019), se detecta que las principales aportaciones se encuentran vinculadas con los estilos de aprendizaje en el entorno de e-learning, los dispositivos móviles y aplicaciones que mejoran 
la educación en ingeniería, los factores críticos de éxito del aprendizaje electrónico y la implementación de modelos para instituciones educativas basados en ecuaciones estructurales.

Estos aspectos muestran que la integración de la gestión del conocimiento en IES está basada en aspectos operativos y técnicos, lo cual solo involucra aspectos del aprendizaje y deja a un lado la gestión estratégica y los flujos información, organizacionales, de decisión, estructurales que, como cualquier organización, están presentes y requieren gestión.

Atendiendo a los resultados que arroja el estudio de la literatura científica surgen las siguientes interrogantes: ¿las IES desarrollan el conocimiento como proceso gerencial?, ¿la gestión del conocimiento en las IES involucra e integra todas las áreas funcionales y sus flujos? En este sentido, se realiza a continuación el diagnóstico de una IES.

\section{Metodología}

La metodología de diagnóstico utilizada para el estudio de las IEs está basada en la propuesta de Alba y Herrera (2014) y Alba (2020), la cual se combina con el modelo de consultoría gerencial definido por Edgar Schein (1990). Según esta, las organizaciones muestran insatisfacciones con el estado actual y los consultores ayudan a identificar problemáticas y generar soluciones.

Bajo la modalidad de intervención-acción se estudió una IES. Este evidenció la necesidad de documentar los procesos informacionales y documentales existentes, y de integrarlos armónicamente con las experiencias y competencias de las personas involucradas. Tuvo como propósito buscar una respuesta al plan de mejoramiento indicado en años anteriores, incorporar nuevos sistemas de información, eliminar errores y pérdidas de información en los procesos, lograr mayor satisfacción de clientes (externos e internos), eliminar la burocracia y las duplicidades, reducir el tiempo total del proceso y obtener un mayor valor agregado. 
Para la aplicación de la metodología se combinaron los siguientes instrumentos: la entrevista, el cuestionario, la observación directa y el análisis documental, que hicieron parte de la metodología propuesta por Alba y Herrera (2014). Asimismo, se aplicaron técnicas grupales como la lluvia de ideas. Para el procesamiento y tabulación de la información se utiliza el software SPSS V25.0. Los resultados de la aplicación de estos instrumentos que se presentan a continuación permiten identificar las problemáticas asociadas a los procesos, la información y el conocimiento.

\section{¿Qué problemáticas se identifican desde la información y el conocimiento en la IES?}

1. No existe un diagrama de procesos en el cual se puedan identificar puntos críticos. Considerando la modalidad de intervención-acción del estudio se construyó el diagrama de flujo de procesos de la Ies en donde aparecen los procesos marcados identificados en cuadros de color azul y de color rosado. Los de color rosado constituyen los puntos críticos o cuellos de botella de esta IEs (figura 2).

2. Existen insatisfacciones en algunos de los procesos definidos para la IES (tabla 1), las cuales se determinan y se les aplica la técnica Ishikawa, lo cual permitió la agrupación de las insatisfacciones por espinas. Se determina que las insatisfacciones presentan correspondencia con las espinas (métodos, infraestructura, recursos materiales y financieros, capital humano). La técnica Ishikawa permitió identificar las causas que en la actualidad presentan mayor afectación para la IEs.

La primera (espina capital humano) causa es que no existe de un canal único de información, por no estar consolidada, guardada y asegurada la información y porque no existe un proceso definido para la entrega. Esto afecta la comunicación, el trabajo grupal y la reserva de la información sensible. En segundo lugar, se evidencian incumplimientos en las matrículas, la solicitud de certificados, la solicitud de conceptos, el envío de material docente y la entrega de notas. 


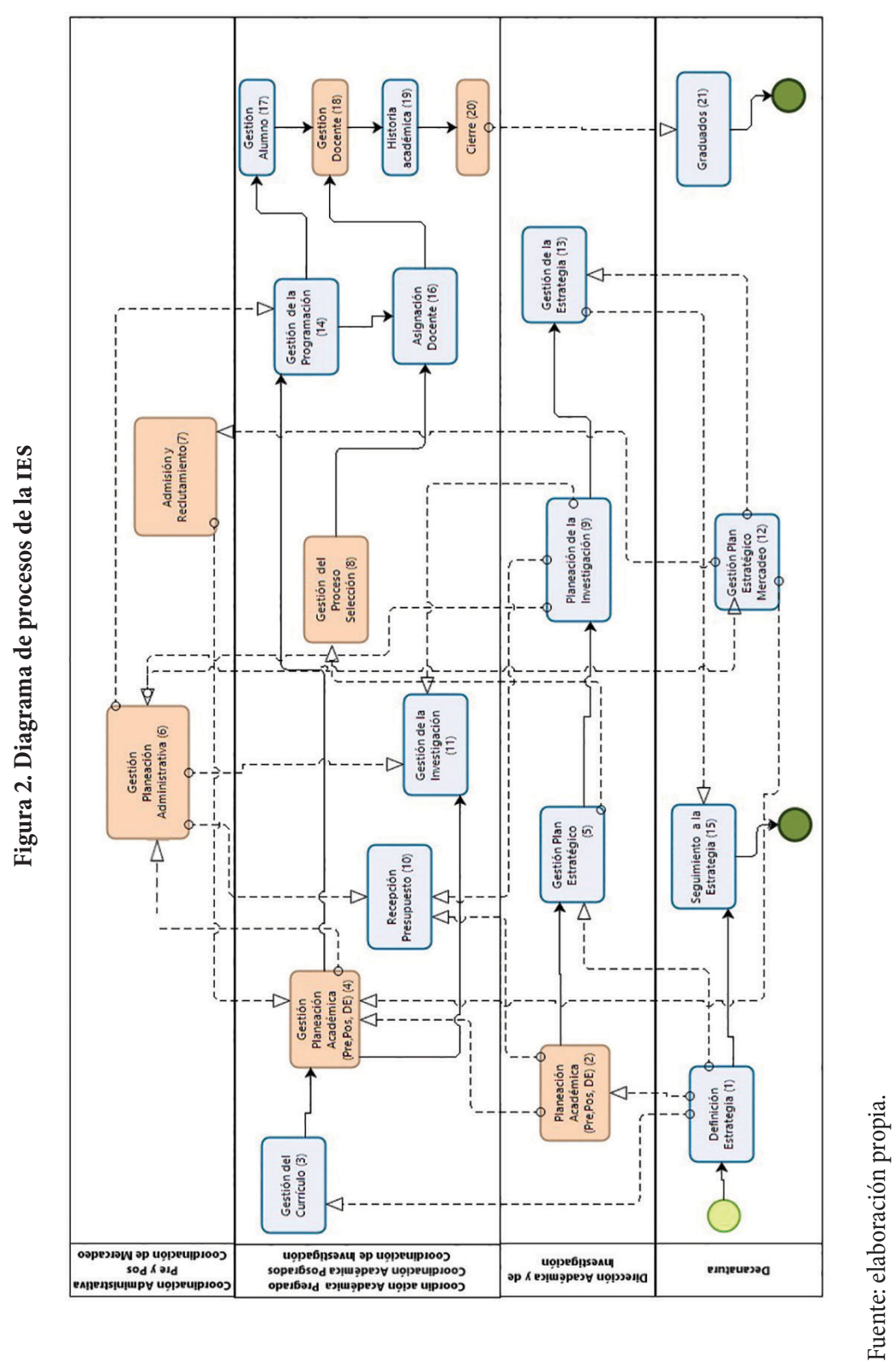


Tabla 1. Agrupación de insatisfacciones por espinas

\begin{tabular}{|c|c|}
\hline Espinas & Insatisfacciones \\
\hline \multirow{7}{*}{ Métodos } & $\begin{array}{l}\text { El número de alumnos con respecto al personal académico } \\
\text { (profesores para clases) constituye cuello de botella } \\
\text { especialmente porque no se tienen los insumos disponibles en } \\
\text { todo momento o no son los idóneos. }\end{array}$ \\
\hline & $\begin{array}{l}\text { La relación docente-estudiante constituye un punto crítico por } \\
\text { la no claridad de los roles, de ahí que no haya incumplimiento } \\
\text { de las responsabilidades (horas de clase y presencia real del } \\
\text { profesor). }\end{array}$ \\
\hline & $\begin{array}{l}\text { No existe proceso de planeación o este no propicia el trabajo } \\
\text { mancomunado de ahí que se evidencie sobrecarga académica. }\end{array}$ \\
\hline & $\begin{array}{l}\text { No se realizan ni se distribuyen a tiempo el material y las } \\
\text { campañas de marketing digital. }\end{array}$ \\
\hline & $\begin{array}{l}\text { No están definidos los puntos estratégicos, lo que dificulta la } \\
\text { planeación de investigación. }\end{array}$ \\
\hline & No hay una orientación real a la estrategia. \\
\hline & No es posible estimar cuántas personas asistirán a grados. \\
\hline \multirow[b]{2}{*}{ Infraestructura } & $\begin{array}{l}\text { Se necesita un sistema de información para la gestión y para los } \\
\text { históricos de pregrado y postgrado. }\end{array}$ \\
\hline & $\begin{array}{l}\text { Los software de aplicación del conocimiento son muy básicos y } \\
\text { no responden al cambio generacional y a todo lo que demanda } \\
\text { el ejercicio. }\end{array}$ \\
\hline $\begin{array}{l}\text { Recursos } \\
\text { materiales y } \\
\text { financieros }\end{array}$ & $\begin{array}{l}\text { En la planeación administrativa y dentro de ella el presupuesto } \\
\text { se ve afectado porque existe variación en lo que se pacta, no se } \\
\text { prevé el pago de tutores y no existe apoyo para las revistas. }\end{array}$ \\
\hline \multirow{3}{*}{ Capital humano } & $\begin{array}{l}\text { La comunicación es poco asertiva, poco profesional e inteligente, } \\
\text { irrespetuosa, no existe trabajo grupal, no une, no es constructiva } \\
\text { y existe poca reserva de la información sensible. }\end{array}$ \\
\hline & $\begin{array}{l}\text { Las matrículas, la solicitud de certificados, la solicitud de } \\
\text { conceptos, el envío de material docente y la entrega de notas en } \\
\text { posgrado no se genera en tiempo. }\end{array}$ \\
\hline & $\begin{array}{l}\text { No hay un canal único de información, la información no está } \\
\text { consolidada, la gente se lleva la consigo por lo que está dispersa } \\
\text { y depende de la voluntad de las personas, no existe un proceso } \\
\text { definido para entregar la información, se evidencia disparidad. }\end{array}$ \\
\hline
\end{tabular}

Fuente: elaboración propia. 
La segunda causa de mayor afectación para la IEs está asociada a la espina. Esta tiene la mayor cantidad de causas asociadas, lo cual permite inferir que las problemáticas de la IEs están muy vinculadas a los métodos o mecanismos de gestión que se utilizan.

La siguiente causa de mayor ponderación por expertos resultóla relación docente-estudiante, por la falta de claridad de los roles, de ahí que exista incumplimiento de las responsabilidades (horas de clase y presencia real del profesor). Por último, pero no menos importante, se descansa en cuarto nivel de afectación la falta de planeación especialmente para la investigación que permita trabajo mancomunado, además de los cumplimientos de compromisos.

3. Se evidenciaron problemas informacionales entre los que destacan:

a. La comunicación se soporta en el correo electrónico, donde no existe criterio de discriminación (prima el criterio de lo enviamos a todos para que todos conozcan).

b. La información se intercambia de un proceso a otro sin recibir tratamiento $o$ análisis.

c. El acceso, localización y socialización de la información es evaluado como regular por el $80 \%$ de los entrevistados.

d. No se evidencia información histórica sobre el comportamiento de estudiantes de pregrado.

e. No existe una plataforma o sistema de información que garantice la toma de decisiones.

f. No se ha realizado un estudio de necesidades informacionales y se evidencia que la información se obtiene por las propias relaciones que establecen los actores sin que medie algún tipo de regulación.

g. La comunicación es valorada como no asertiva de que ahí, que se evidencia en un $90 \%$ insatisfacción. Dentro de las afectaciones que 
provocan los problemas de comunicación se encuentran: la no reserva de información con la parte de docente, estudiante y facultad. Los problemas personales que afectan el clima organizacional, la falta de responsabilidad en las tareas que se entregan y el no reconocimiento de los niveles de dirección.

4. Lagestión del conocimiento se está afectando porque no todo lo que se adquiere se guarda, se transfiere y es utilizado e incorporado en el diseño de nuevas acciones o mejoras para la IES, por lo que parte del conocimiento adquirido se estaría perdiendo (figura 3 y 4 ).

En esta etapa de la metodología de diagnóstico se aplica la técnica del cuestionario (anexo 1) a un total de 10 directivos. El procesamiento del cuestionario (anexo 1) por medio del software SPSS V25.0 con el empleo de diagramas de cajas (figura 3 y 4 ) permitió evidenciar que, según se constata en la figura 3, la adquisición es evaluada con una dispersión común que ubica su frecuencia de utilización en la escala más alta (entre frecuente y muy frecuente). Con respecto al almacenamiento, existe una dispersión de la respuesta entre 1 y 2 (de poco a frecuente). Por otra parte, al igual que el almacenamiento, la transferencia es un proceso que se utiliza entre poco $y$ frecuentemente, por lo que presenta una dispersión entre 1 y 2. En el caso del proceso de uso se evidencia que se utiliza frecuentemente, es decir, más del $50 \%$ de los encuestados lo consideró así; sin embargo, existen dos casos atípicos en 1 y 3. Por último, y en el proceso de creación, se evidencia una dispersión entre 1 y 2, lo cual permite inferir que este proceso se utiliza entre poco y frecuentemente.

Si se analiza de manera integral la figura 3, llama la atención la frecuencia otorgada a las acciones de almacenamiento, transferencia, uso y creación pues, al no estar en el orden de la adquisición, se puede inferir que todo lo que se adquiera no va a poder ser guardado, transferido, utilizado e incorporado en el diseño de nuevas acciones o mejoras, por lo que parte del conocimiento adquirido se estaría perdiendo. 
Figura 3. Frecuencia de las etapas de gestión del conocimiento en la IES

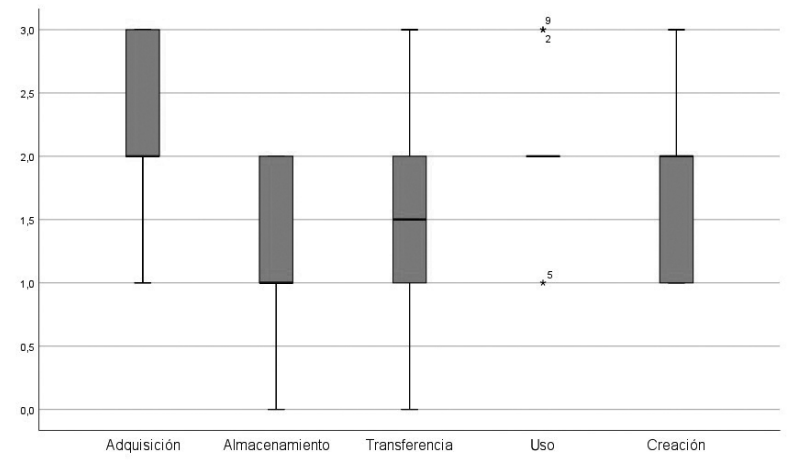

Fuente: elaboración propia.

En cuanto al nivel de importancia (figura 4) que se le confiere a cada etapa del proceso de gestión del conocimiento, se pudo evidenciar que la adquisición es evaluada con una dispersión entre 4 y 5 , siendo 5 el valor que más se repite por los expertos en este criterio. El proceso de almacenamiento posee una dispersión entre 1 y 2 , que lo ubica en el último nivel de importancia. La transferencia es ubicada en 3 por el $50 \%$ de los expertos; sin embargo, existen dos casos atípicos que la ubican en 1,2,5. El proceso de uso presenta una dispersión entre 3 y 5 , lo cual lo ubica en el segundo orden de prioridad y, por último, la creación se encuentra entre 2 y 3 , siendo 2 el valor que más se repite por expertos.

$\mathrm{Al}$ analizar estas categorías (figura 4), se observa que se evalúa a la adquisición en primer orden de prioridad con existiendo total consenso; sin embargo, al conferirle el valor más bajo al proceso de almacenamiento, se infiere que no todos los datos adquiridos se logran almacenar por lo que en alguna medida se verá afectado el ciclo completo de gestión del conocimiento.

Por otra parte, resulta coherente la evaluación obtenida en los procesos de almacenamiento, transferencia y uso. Sin embargo, mientras que 
los procesos de almacenamiento y creación cuenten con un valor más bajo de importancia, se infiere que no se realiza innovación y el proceso de creación se ve afectado y no incorporado en los nuevos programas, acciones y mejoras que les permitan destacarse en el mercado. Por medio de la integración de ambos análisis, se conoce que los procesos de almacenamiento y creación no son priorizados, por lo que no se realizan con mucha frecuencia.

Figura 4. Importancia de las etapas de gestión del conocimiento en la IES

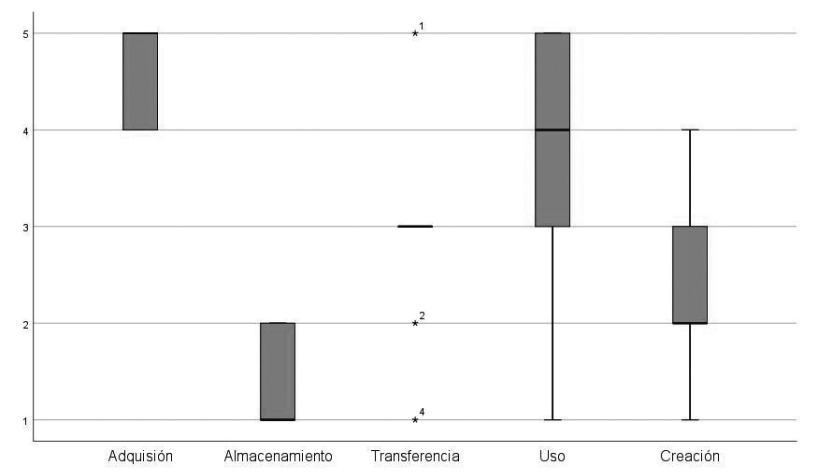

Fuente: elaboración propia.

5. Posibilidad de crecimiento en investigación, mercadeo, idioma inglés, liderazgo situacional, presupuesto, contratación, admisiones y reclutamiento, y gestión de la planeación administrativa y gestión académica. Estas posibilidades de crecimiento se obtuvieron por medio de la técnica de entrevista personal, en donde fueron detectadas como necesidades por los propios entrevistados. 


\section{Resultados}

\section{¿Cómo mejorar los procesos informacionales y de conocimiento en la IES?}

Para la mejora se propone aplicar la concepción del infoconocimiento en un modelo de gestión para la IEs, lo cual permite articular la gestión de los procesos, la integración de los flujos de información y conocimiento entre los actores, sus experiencias, el aprendizaje y las competencias, a partir del desarrollo de un pensamiento en espiral como enfoque de proceso, creciente y sinérgico. En consecuencia, se agrega valor y crea las bases para la innovación organizacional y de productos (figura 5).

La explicación de la figura 4 inicia de izquierda a derecha; comienza por la identificación de las etapas por las que transita la IEs. Se detecta una etapa gerencial (marcada en la figura 5 con imagen de barras), donde se articulan los siguientes elementos: definición de la estrategia, seguimiento a la estrategia, gestión plan estratégico mercado, graduados, planeación académica, gestión plan estratégico, planeación de la investigación, gestión de la estrategia.

En el nivel táctico de dirección (marcado en la figura 5 con imagen de piñones de engranaje), se identificó la gestión de la investigación, gestión del currículo, gestión planeación académica, recepción del presupuesto, gestión del proceso de selección, gestión de la programación, asignación docente, gestión docente y del alumno, historia académica, cierre. Por último, en el nivel operativo (marcada en la figura 5 con imagen de carrito de mercado) se ubicaron a la gestión de la planeación administrativa y admisiones y reclutamiento.

Estos elementos ingresan a la espiral (infoconocimiento), la cual permite la determinación y organización de la información relevante mediante el filtrado, la disponibilidad de los contenidos, el almacenamiento de fuentes y la incorporación de juicios de valor en puntos clave de la IES, aportados 
por la participación de expertos y personal involucrado, que a su vez intervienen de forma creadora en la adquisición, la comunicación y la toma de decisiones.

Las aplicaciones, socialización y registro de las decisiones tomadas alimentan la espiral, lo que permite generar valor a las actividades y procesos, así como promover la innovación o mejora continua que se obtiene como resultado de los continuos ciclos de la espiral. Los resultados de estos procesos se socializan como vías para promover la colaboración, apoyados en plataformas y mecanismos informáticos que permiten acceder a los resultados y colaborar para generar transformaciones con valor agregado mediante la transferencia, cuyos resultados se ubican en repositorios que permiten su localización y realimentación, aspectos que son monitoreados y administrados.

El éxito de la espiral, es decir de los elementos marcados en cursiva en los dos párrafos anteriores, se basa en un conjunto de herramientas que articuladas en un enterprise information portal (EIP) permiten responder a las demandas de cada punto de la espiral (los elementos marcados en cursiva). Entre las herramientas que formarán parte del EIP están las siguientes:

- El observatorio se encargará de la vigilancia del entorno científico sobre la profesión y servirá para generar nuevos conocimientos a la IEs. Establecerá los vínculos entre las IEs (nacionales e internacionales) con vistas a compartir información y entregar productos, información pertinente y actualizada que permitan a los directivos e investigadores identificar los riesgos y anticiparse a las oportunidades. Por medio de la vigilancia, se estará analizando temas con la participación de expertos; a través de esta tecnología de información, se promueve la reflexión y el intercambio de conocimiento en la red. 
La gestión del infoconocimiento en instituciones

de educación superior

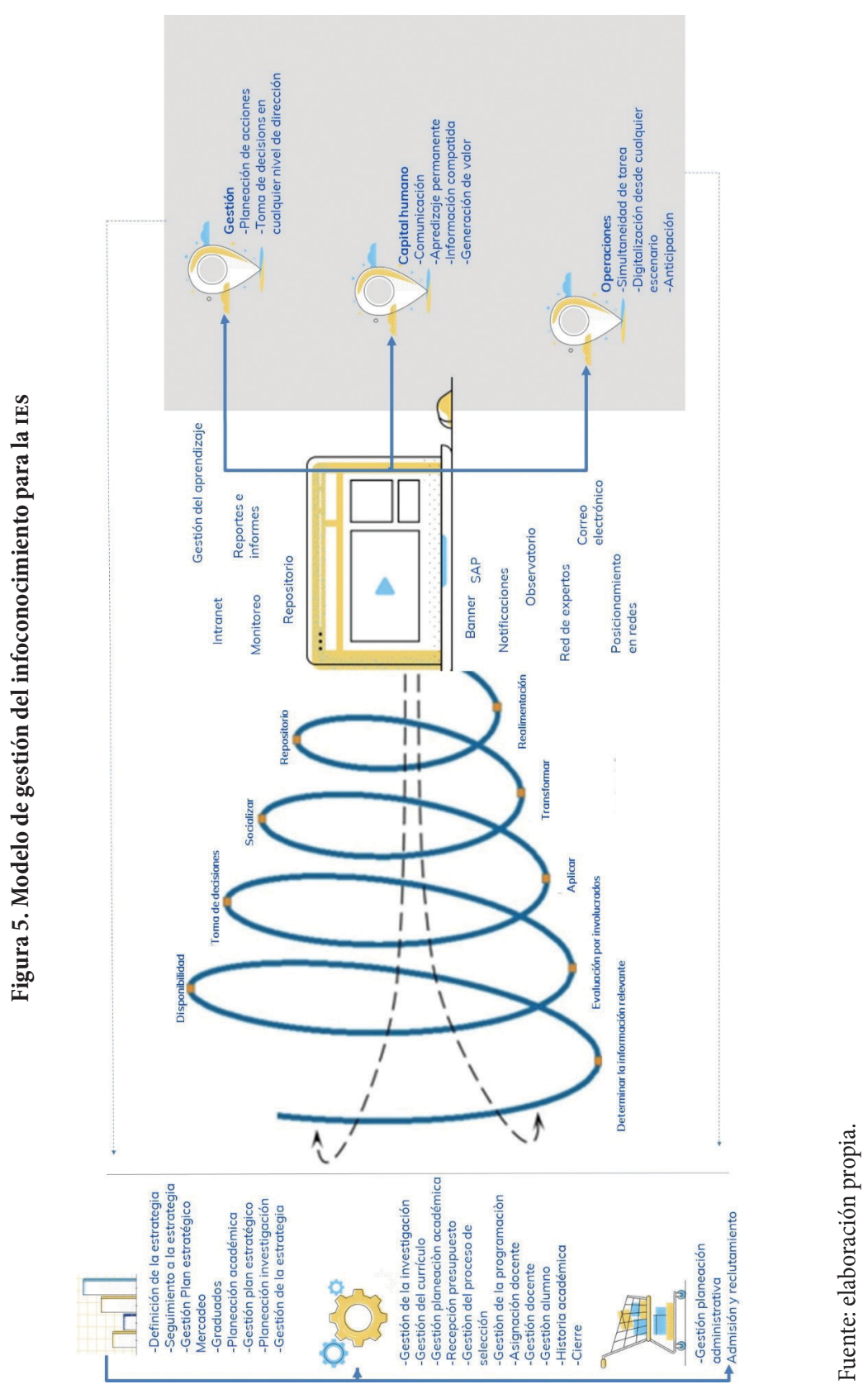


- El bloque de notificaciones se encargará de mantener el proceso comunicativo constante y sentar las bases primarias de la comunicación interna, a través de avisos, alarmas y mensajes que llegan a los escenarios de cada directivo o especialista indicándoles fechas de vencimiento, actualizaciones, nuevas informaciones, nuevas asignaciones de trabajo y un conjunto de acciones que son configurables de acuerdo con las exigencias de la dirección, como son avisos o mensajes de reconocimiento, retrasos e incumplimientos.

- El posicionamiento en redes sociales permite realizar seguimientos de clientes, identificar las estrategias de la competencia, anticiparse a los avances tecnológicos, identificar las necesidades sobre nuevos territorios para innovar y contribuir a la inteligencia del negocio y a los estudios de mercado que actualmente se realizan.

- La gestión del aprendizaje se basa en la generación de espacios de aprendizaje, los cuales, enlazados con las posibilidades de crecimiento, permitirán mantener actualizado la base de conocimiento tácito de la IES.

- Los repositorios garantizarán el almacenamiento y conservación del conocimiento explícito, lo que permitirá realizar análisis históricos de la IEs. Se propone mantener la utilización de estos tres tipos de repositorios y se sugiere se empleen de la siguiente forma:

- Sharepoint para la gestión histórica de los procesos generales de la IES.

- SAP para manejo histórico de los estudiantes de pregrado.

- Banner para los estudiantes de posgrado. Es importante destacar que los reportes e informes que se obtengan de este repositorio y de SAP se ubicarán en el Sharepoint para garantizar acciones de control y toma de decisiones en el nivel estratégico. 
- La red de expertos estará funcionando continuamente para las acciones de estrategia de la IES, plan de mejoramiento, acreditación, estrategia de investigación, estudios de mercado y las OPL.

- El monitoreo se establece para conocer permanentemente los cursos promocionados frente al cursos impartidos; el comportamiento de estudiantes frente a plan de graduados, y los estudiantes inscritos frente a los estudiantes pagados, los próximos cursos, alianzas, temas de interés mensual, el estado de cada actividad posgraduada (especialización, maestrías) por tipo, materia, próximas acciones, fecha de cierre, necesidades y eventualidades. El reporte de la planeación académica, reporte de egresos, solicitudes (tiquetes, transporte, alojamiento), rubros presupuestados frente a los gastados y el seguimiento al cumplimiento de planes de trabajo.

- La intranet debe recoger la información de interés de la IES, por ejemplo: la estrategia de la IES, la estrategia de investigación, los objetivos estratégicos, la planeación de investigación formativa, entre otras actividades que pongan en conocimiento a la IES en determinadas cuestiones.

- El correo electrónico deberá funcionar para las comunicaciones externas principalmente; para la comunicación interna por correo electrónico, se debe considerar la descripción de procesos que aporta este estudio, con el objetivo de evitar sobrecarga de correos.

Este modelo finaliza con el impacta en 3 áreas: la gestión, el capital humano y las operaciones de la IEs. En la gestión resulta relevante su contribución a la planificación de las acciones de la IEs, toda vez que las entradas de los datos directivos son monitoreados y compartidos por cada actor de la organización según sus funciones, de manera que se dota al proceso de una mayor objetividad en los aspectos estratégicos, reales y previsibles. Dentro del ámbito de la gestión, el modelo también promueve la posibilidad de la toma de decisiones oportunas, al brindar de forma actualizada y sistemática la información necesaria y precisa que refuerza la articulación entre cada 
área y proceso de la organización. Asociado a la toma de decisiones y los recursos, el modelo promueve el desarrollo de la colaboración con un espíritu anticipador, lo cual pretende ofrecer soluciones a posibles problemas para minimizar su magnitud.

Otra área en la que se obtienen resultados es en el desarrollo del capital humano, toda vez que el modelo incide en el desarrollo de capacidades, el aprendizaje, la comunicación, la colaboración y socialización permanente. Además, permite la detección y reconocimiento de expertos, la capacitación y entrenamiento de directivos, especialistas y trabajadores, y el desarrollo de competencias necesarias, así como la creación y fomento de espacios de conocimientos e intercambios de experiencias.

Vinculado con las operaciones, el modelo asegura la mayor conectividad entre actores y procesos. Logra la simultaneidad de las tareas y la reducción de desconexiones entre procesos y actividades, ya que su diseño asume el planteamiento de flujos de operaciones en los cuales, desde diferentes escenarios, se podrá digitar (captar), procesar y mostrar información, ejecutar acciones y obtener realimentación de las decisiones tomadas. Lo anterior constituye un mecanismo de control, pues al estandarizar las entradas y registros de información, su conservación y circulación, contribuye a la confiabilidad, al disminuir los errores de usuarios y los fraudes.

Esta modelación teórica establece dos retroalimentaciones: una de mantenimiento de la organización y otra de mantenimiento del EIP. La primera se da en la revisión de los resultados obtenidos (gestión, capital humano y operaciones) para el perfeccionamiento de las condiciones de entrada y funcionamiento de los elementos de la IEs; la segunda permite la actualización del EIP, lo cual significa que las salidas que estas ofrecen tributan al funcionamiento, pertinencia y desarrollo de la espiral de infoconocimiento. Esto genera valor en la IEs, ya que, cuando se produce un nuevo registro, se realiza un crecimiento de las acciones de información y conocimiento, que se mueven dentro de la espiral; en consecuencia, se logra incrementar la pertinencia y efectividad en las futuras consultas y operaciones de gestión. 


\section{¿Qué resuelve el modelo de gestión del infoconocimiento?}

Esta propuesta se encarga de responder a las insatisfacciones definidas en la tabla 1 desde la información y el conocimiento. Para esta investigación se clasifican estas insatisfacciones en las salidas que ofrece la gestión del infoconocimiento, de manera que puede detectar una salida hacia el capital humano ( $\mathrm{CH}$ ), la gestión y las operaciones (tabla 2).

Tabla 2. Insatisfacciones y problemas para solucionar desde el infoconocimiento

\begin{tabular}{|l|l|l|}
\hline \multicolumn{1}{|c|}{$\begin{array}{c}\text { Insatisfacciones y problemas } \\
\text { detectados }\end{array}$} & $\begin{array}{c}\text { Temas en los } \\
\text { cuales trabajar }\end{array}$ & Infoconocimiento \\
\hline $\begin{array}{l}\text { No existe un canal único de } \\
\text { información, la información no } \\
\text { está consolidada, la gente se lleva } \\
\text { la información, información } \\
\text { dispersa depende de la voluntad } \\
\text { de las personas, no existe un } \\
\text { proceso definido para entregar la } \\
\text { información, se evidencia disparidad } \\
\text { de la información. }\end{array}$ & $\begin{array}{l}\text { Información } \\
\text { dispersa, } \\
\text { disparidad, entrega } \\
\text { de información }\end{array}$ & CH \\
\hline $\begin{array}{l}\text { No están definidos los puntos } \\
\text { estratégicos lo que dificulta la } \\
\text { planeación de investigación. }\end{array}$ & Planeación & Gestión \\
\hline $\begin{array}{l}\text { Se evidencia desconocimiento en el } \\
\text { misión, visión y objetivos estratégicos } \\
\text { por opL sin seguimiento. }\end{array}$ & $\begin{array}{l}\text { Desconocimiento } \\
\text { de planeación } \\
\text { estratégica }\end{array}$ & Gestión \\
\hline $\begin{array}{l}\text { La comunicación se soporta en el } \\
\text { correo electrónico, donde no existe } \\
\text { criterio de discriminación (prima el } \\
\text { criterio de lo enviamos a todos para } \\
\text { que todos conozcan). }\end{array}$ & $\begin{array}{l}\text { Discriminación de } \\
\text { la información }\end{array}$ & CH, operaciones \\
\hline $\begin{array}{l}\text { La información se intercambia de un } \\
\text { proceso a otro sin recibir tratamiento } \\
\text { o análisis. }\end{array}$ & $\begin{array}{l}\text { Tratamiento, } \\
\text { análisis } \\
\text { informacional }\end{array}$ & CH, operaciones \\
\hline $\begin{array}{l}\text { Dentro de las Tic que soportan el } \\
\text { trabajo se evidencia conocimiento de } \\
\text { la suite de Office la cual no se utiliza. }\end{array}$ & $\begin{array}{l}\text { Aprendizaje y } \\
\text { capacitación }\end{array}$ & CH \\
\hline
\end{tabular}




\begin{tabular}{|c|c|c|}
\hline $\begin{array}{c}\text { Insatisfacciones y problemas } \\
\text { detectados }\end{array}$ & $\begin{array}{l}\text { Temas en los } \\
\text { cuales trabajar }\end{array}$ & Infoconocimiento \\
\hline $\begin{array}{l}\text { El acceso, localización y socialización } \\
\text { de la información es evaluado } \\
\text { como regular por el } 80 \% \text { de los } \\
\text { entrevistados. }\end{array}$ & Organización & $\mathrm{CH}$ \\
\hline $\begin{array}{l}\text { Se evidencia ausencia de información } \\
\text { histórica sobre el comportamiento de } \\
\text { estudiantes de pregrado. }\end{array}$ & $\begin{array}{l}\text { Almacenamiento } \\
\text { de información }\end{array}$ & $\mathrm{CH}$ \\
\hline $\begin{array}{l}\text { No existe una plataforma o sistema } \\
\text { de información que garantice la toma } \\
\text { de decisiones. }\end{array}$ & Toma de decisiones & Gestión \\
\hline $\begin{array}{l}\text { No se ha realizado un estudio de } \\
\text { necesidades informacionales y se } \\
\text { evidencia que la información se } \\
\text { obtiene por las propias relaciones que } \\
\text { establecen los actores sin que medie } \\
\text { algún tipo de regulación. }\end{array}$ & $\begin{array}{l}\text { Flujo de } \\
\text { información, } \\
\text { documento que } \\
\text { regule intercambio }\end{array}$ & $\mathrm{CH}$ \\
\hline $\begin{array}{l}\text { Falta capacitación para asumir } \\
\text { responsabilidades. }\end{array}$ & Aprendizaje & $\mathrm{CH}$ \\
\hline $\begin{array}{l}\text { No existe un documento que regule } \\
\text { el intercambio de información y los } \\
\text { tiempos de respuesta. }\end{array}$ & $\begin{array}{l}\text { Intercambio de } \\
\text { información, } \\
\text { tiempos de } \\
\text { respuesta }\end{array}$ & $\mathrm{CH}$, operaciones \\
\hline $\begin{array}{l}\text { La comunicación es valorada como } \\
\text { no asertiva; de ahí se evidencia } \\
\text { un } 90 \% \text { insatisfacción. Dentro } \\
\text { de las afectaciones que provocan } \\
\text { los problemas de comunicación } \\
\text { se encuentran la no reserva de } \\
\text { información con la parte de } \\
\text { docente, estudiante y facultad; los } \\
\text { problemas personales que afectan } \\
\text { el clima organizacional; la falta de } \\
\text { responsabilidad en las tareas que se } \\
\text { entregan y el no reconocimiento de } \\
\text { los niveles de dirección. }\end{array}$ & $\begin{array}{l}\text { Gestión de } \\
\text { información, } \\
\text { comunicación, } \\
\text { información } \\
\text { compartida }\end{array}$ & $\mathrm{CH}$ \\
\hline
\end{tabular}

Fuente: elaboración propia. 


\section{¿Cómo poner en práctica el modelo de gestión del infoconocimiento para la IES?}

Para el desarrollo del modelo de gestión del infoconocimiento en la IEs se definió un procedimiento compuesto por 2 etapas (ejecución y control) y 8 pasos (figura 6).

Figura 6. Procedimiento de aplicación del modelo de infoconocimiento.

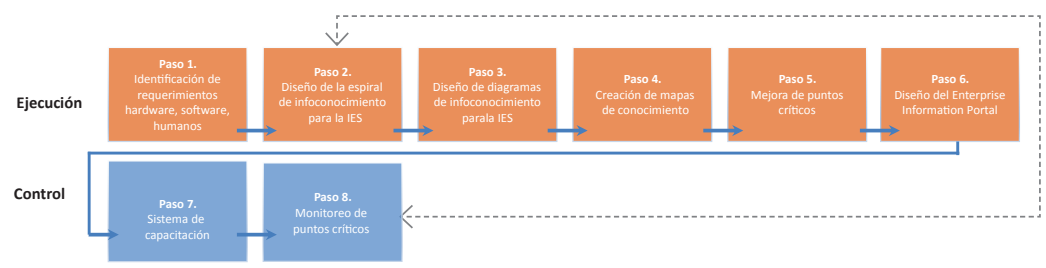

Fuente: elaboración propia.

\section{Conclusiones}

La información, el conocimiento y su gestión se han convertido en una opción estratégica de cualquier tipo de organización para alcanzar los objetivos y metas. Es por ello que, en el contexto de las IEs, estos se convierten en el elemento primordial para actuar. Sin embargo, la gestión del conocimiento en estas instituciones sigue siendo imprecisa y deja a la espontaneidad un proceso que debería ser planificado, organizado y controlado. Estos aspectos muestran que integrar la gestión del conocimiento en IEs vincula aspectos operativos y técnicos, especialmente todo lo relacionado con el aprendizaje, y deja a un lado la gestión estratégica y los flujos de información, organizacionales, de decisión, estructurales que, como cualquier organización, están presentes y requieren gestión.

La metodología de diagnóstico mediante la modalidad de intervenciónacción permitió no solo conocer el estado de los procesos informacionales y de conocimiento, sino también construir el mapa de procesos de la IES 
y detectar puntos críticos que dilatan el ciclo normal de las acciones que se llevan a cabo. También, un conjunto de insatisfacciones que agrupadas en espinas permite detectar que la problemática central de esta IES está asociada a los mecanismos de gestión que se utilizan, especialmente por la relación docente-estudiante, por la no claridad de los roles y por el incumplimiento de las responsabilidades. Asimismo, se reflejan problemas comunicacionales, informacionales y de conocimiento. Estos aspectos unidos a la carencia reflejada en la literatura científica llevaron a proponer la implementación del modelo de gestión del infoconocimiento.

El modelo en esta IES permite articular la gestión de los procesos, la integración de los flujos de información y conocimiento entre los actores, sus experiencias, el aprendizaje y las competencias, a partir del desarrollo de un pensamiento en espiral como enfoque de proceso, creciente y sinérgico, que agrega valor y crea las bases para la innovación organizacional y de productos. Lo anterior permite resolver las problemáticas antes identificadas y cumplir con el objetivo general de esta investigación. Para su puesta en marcha, se creó un procedimiento compuesto por 2 etapas (ejecución y control) y 8 pasos los que articulados contribuyen a resolver las problemáticas y puntos críticos de la IEs.

\section{Referencias}

Alba, M. (2020). El infoconocimiento: una propuesta gerencial. Bogotá: Universidad Externado de Colombia.

Alba, M. y Herrera, K. (2014). Metodología para el diagnóstico de la información y el conocimiento en cadenas de suministro. Revista Cubana de Contabilidad y Finanzas, Cofin Habana, (2), 11-19.

Alba, M. y Herrera, K. C. (2016). Modelo de gestión del infoconocimiento para cadenas de suministro de ron a granel. Cofin Habana, 10(1), 28-38. Consultado en http://scielo.sld.cu/scielo.php?script=sci_abstract\&pid=\$207360612016000100003\&lng=pt\&nrm=iso 
Alhabeeb, A. y Rowley, J. (2018). E-learning critical success factors: Comparing perspectives from academic staff and students. Computers and Education, 127, 1-12. DoI: https://doi.org/10.1016/j.compedu.2018.08.007

Angulo, E. y Negrón, M. (2008). Modelo holístico para la gestión del conocimiento. Negotium: revista de ciencias gerenciales, 4(11), 38-51.

Caicedo, J. C. F. (2010). La gestión del conocimiento y las herramientas colaborativas: una alternativa de aplicación en Instituciones de educación superior. Revista de Investigación, 34(71), 11-31. Consultado en https://dialnet.unirioja. es/servlet/articulo? codigo $=3704580$

De Freitas, V. y Oltra, G. Y. (2014). Modelo holístico de sistema de gestión del conocimiento para las instituciones de educación superior. Enl@ce. Revista Venezolana de Información, Tecnología y Conocimiento, 11(3), 4. Consultado en https://dialnet.unirioja.es/servlet/articulo?codigo $=5101931$

Doulik, P., Skoda, J. y Simonova, I. (2017). Learning styles in the e-learning environment: The approaches and research on longitudinal changes. International Journal of Distance Education Technologies, 15(2), 45-61. DoI: https://doi. org/10.4018/IJDET.2017040104

Gafurov, I. R., Safiullin, M. R., Akhmetshin, E. M., Gapsalamov, A. R. y Vasilev, V. L. (2020). Change of the higher education paradigm in the context of digital transformation: From resource management to access control. International Journal of Higher Education, 9(3), 71-85.Dor: https://doi.org/10.5430/ijhe. v9n3p71

García-Peñalvo, F. J. (2016). La socialización como proceso clave en la gestión del conocimiento. Education in the Knowledge Society, 17(2), 7-14. DoI: http:// dx.doi.org/10.14201/eks2016172714

Martínez Villalba, J. A., y Sánchez Muñoz, S. (2018). Generación de competencias con base en la gestión de conocimiento científico. REICE. Revista Iberoamericana sobre Calidad, Eficacia y Cambio en Educación, 16(2), 61-76. Consultado en https://revistas.uam.es/reice/article/view/9392/9608 
Pal Pandi, A., Paranitharan, K. P. y Jeyathilagar, D. (2018). Implementation of IEQMS model in engineering educational institutions - a structural equation modelling approach. Total Quality Management and Business Excellence, 29 (1-2), 29-57. Dor: https://doi.org/10.1080/14783363.2016.1154431

Safiullin, M. R. y Akhmetshin, E. M. (2019). Digital transformation of a university as a factor of ensuring its competitiveness. International Journal of Engineering and Advanced Technology, 9(1), 7387-7390. Dor: https://doi.org/10.35940/ ijeat.A3097.109119

Schein, E. H. (1990). Consultoría de procesos (vol. 2). Addison-Wesley Iberoamericana.

Simonova, I. y Poulova, P. (2016, 24-26 de febrero). Mobile devices and applications enhancing engineering education in ESP [ponencia]. Proceedings of 201613 th International Conference on Remote Engineering and Virtual Instrumentation. IEEE Madrid, España. Dor:10.1109/REV.2016.7444458

Topete, C., Bustos, E. y Bustillos, E. S. (2012). Gestión del conocimiento para promover la productividad académica de los institutos tecnológicos en la sociedad del conocimiento. Sinéctica, (38), 1-15. Consultado en http://www. scielo.org.mx/scielo.php?script=sci_arttext\&pid=S1665-109X2012000100005 


\section{Anexos}

\section{Anexo 1. Cuestionario. Valoración de los criterios de gestión del conocimiento y su frecuencia (Cuesta y Valencia, 2014)}

\section{Estimado directivo}

Con vistas a conocer como es el proceso de gestión del conocimiento en su organización se le pide su evaluación sobre los criterios que se adjuntan.

\begin{tabular}{|c|c|c|}
\hline \multirow{2}{*}{$\begin{array}{l}\text { Criterios de gestión del } \\
\text { conocimiento }\end{array}$} & $\begin{array}{c}\text { Importancia del } \\
\text { criterio }^{4}\end{array}$ & $\begin{array}{l}\text { Frecuencia con } \\
\text { la cual se hace }\end{array}$ \\
\hline & $1-5$ & $\begin{array}{llll}0 & 1 & 2 & 3\end{array}$ \\
\hline $\begin{array}{l}\text { 1. Adquisición. ¿Los miembros de la } \\
\text { organización son capaces de adquirir } \\
\text { nuevos conocimientos para cumplir } \\
\text { sus funciones? }\end{array}$ & & \\
\hline $\begin{array}{l}\text { 2. Almacenamiento. ¿Los miembros } \\
\text { de la organización documentan y } \\
\text { guardan el conocimiento que poseen? }\end{array}$ & & \\
\hline $\begin{array}{l}\text { 3. Transferencia. ¿Los miembros } \\
\text { de la organización comparten el } \\
\text { conocimiento que poseen con los } \\
\text { demás miembros? }\end{array}$ & & \\
\hline $\begin{array}{l}\text { 4. Uso. ¿Los miembros de la } \\
\text { organización aplican y utilizan el } \\
\text { conocimiento adquirido para realizar } \\
\text { las funciones? }\end{array}$ & & \\
\hline
\end{tabular}

4 Indica el grado de importancia que tiene cada criterio estratégico para los responsables de la organización, considerando que 1 representa el valor más bajo y 5 representa el valor más alto y no puede haber criterios con el mismo valor de importancia.

5 Frecuencia con la que se hace $(0,1,2,3)$ representa (ninguna, poca, frecuente, mucho). 


\begin{tabular}{|l|c|c|}
\hline \multirow{2}{*}{$\begin{array}{c}\text { Criterios de gestión del } \\
\text { conocimiento }\end{array}$} & $\begin{array}{c}\text { Importancia del } \\
\text { criterio }^{4}\end{array}$ & $\begin{array}{c}\text { Frecuencia con } \\
\text { la cual se hace }\end{array}$ \\
\cline { 2 - 4 } & $\mathbf{1 - 5}$ & $\mathbf{0} \quad \mathbf{1} \quad \mathbf{2} \mathbf{3}$ \\
\hline $\begin{array}{l}\text { 5. Creación. ¿Los miembros de la } \\
\text { organización generan nuevos } \\
\text { conocimientos, experiencias, ideas e } \\
\text { innovaciones como resultado de su } \\
\text { trabajo? }\end{array}$ & \\
\hline $\begin{array}{l}\text { 6. ¿En qué momento la información y el conocimiento no han estado presentes en la } \\
\text { actividad que usted dirige que ha ocasionado problemas? }\end{array}$ \\
\hline \\
\hline
\end{tabular}

Gracias por su colaboración. 